\title{
A HOMEOMORPHISM ON $s$ NOT CONJUGATE TO AN EXTENDABLE HOMEOMORPHISM
}

\author{
JAN VAN MILL
}

(Communicated by James E. West)

Dedicated to Profesor Yukihiro Kodama on his sixtieth birthday

\begin{abstract}
Consider $s=\prod_{i=1}^{\infty}(-1,1)_{i}$ and its compactification $Q=$ $\prod_{i=1}^{\infty}[-1,1]_{i}$. Anderson and Bing asked whether for every homeomorphism $f: s \rightarrow s$ there is a homeomorphism $\phi: s \rightarrow s$ such that $\phi^{-1} f \phi$ is extendable to a homeomorphism $\overline{\phi^{-1} f \phi}: Q \rightarrow Q$. The aim of this note is to construct a counterexample to this question.
\end{abstract}

\section{INTRODUCTION}

Consider $s=\prod_{i=1}^{\infty}(-1,1)_{i}$ and its compactification

$$
Q=\prod_{i=1}^{\infty}[-1,1]_{i}
$$

A homeomorphism $f: s \rightarrow s$ is said to be conjugate to an extendable homeomorphism if there is a homeomorphism $\phi: s \rightarrow s$ such that $\phi^{-1} f \phi$ is extendable to a homeomorphism $\overline{\phi^{-1} f \phi}: Q \rightarrow Q$. In [1], Anderson and Bing asked whether every homeomorphism on $s$ is conjugate to an extendable homeomorphism. Sakai and Wong [5] recently presented several conditions that imply that a homeomorphism on $s$ satisfying one of them is conjugate to an extendable homeomorphism.

The corresponding finite-dimensional problem, replacing $s$ by $\left\{x \in \mathbf{R}^{n}:\|x\|\right.$ $<1\}$ and $Q$ by $\left\{x \in \mathbf{R}^{n}:\|x\| \leq 1\right\}$, has a simple negative answer for every $n \geq 2$. For details, see [5]. We remark that Eric van Douwen communicated a similar solution to us at least 5 years ago.

Received by the editors March 18, 1988.

1980 Mathematics Subject Classification (1985 Revision). Primary 57N20.

Key words and phrases. Hilbert cube, isotopy, conjugation, capset.

This note was written during a visit to Japan in February 1988. I am indebted to the Department of Mathematics of the University of Tsukuba for generous hospitality and support. 
The aim of this note is to present an example of a homeomorphism on $s$ which is not conjugate to an extendable homeomorphism, thereby answering the Anderson and Bing question in the negative.

\section{THE CONSTRUCTION}

Let $X$ be a space. An isotopy on $X$ is a homotopy $H: X \times I \rightarrow X$ such that the function $\phi: X \times I \rightarrow X \times I$ defined by

$$
\phi(x, t)=(H(x, t), t), \quad(x \in X, t \in I)
$$

is a homeomorphism. Observe that if $H: X \times I \rightarrow X$ is an isotopy then for every $t \in I$ the function $H_{t}: X \rightarrow X$ defined by

$$
H_{t}(x)=H(x, t), \quad(x \in X)
$$

is a homeomorphism.

A Hilbert cube is a space homeomorphic to $Q$. Let $M$ be a Hilbert cube. A capset of $M$ is a subset $A \subseteq M$ for which there is a homeomorphism $f: M \rightarrow Q$ such that $f(A)=Q \backslash s$. In addition, a closed set $B$ of $M$ is called a $Z$-set if for every $\varepsilon>0$ there is a map $f: M \rightarrow M \backslash B$ with $d\left(f, 1_{M}\right)<\varepsilon$ ( $1_{X}$ means the identity function on $X$ ). It is known that if $A \subseteq M$ is a capset and $B \subseteq M$ is a $Z$-set then $A \backslash B$ is a capset (for details see [2] and [3]).

We shall now describe a special isotopy on $s$ that shall be important later in the construction of the example.

2.1. Proposition. There are an isotopy $H: s \times I \rightarrow s$ on $s$ and two compact sets $A, B \subseteq s$ such that

(1) $H_{0}=1_{s}$;

(2) if $E \subseteq s$ is closed and misses $A$ and if $U$ is a neighborhood of $B$ in $s$ then there is an $n \in \mathbf{N}$ such that $\left(H_{1}\right)^{n}(E) \subseteq U$.

Proof. There is an isotopy $F: I \times I \rightarrow I$ such that

(3) $F_{0}=1_{I}$,

(4) for every $t \in I, F_{t}(0)=0$ and $F_{t}(1)=1$,

(5) $\forall t \in(0,1], \lim _{n \rightarrow \infty}\left(F_{1}\right)^{n}(t)=1$.

For example, $F$ can be defined by $F(s, t)=(1-t) s+t \sqrt{s}$.

Put $\vec{Q}=Q \times I$. The isotopy $F$ induces an isotopy $G: \vec{Q} \times I \rightarrow \vec{Q}$ as follows:

$$
G((x, s), t)=\left(x, F_{t}(s)\right), \quad(x \in Q, t, s \in I) .
$$

Since $Q \times\{0,1\}$ is a $Z$-set in $\vec{Q}$ and $\vec{Q} \backslash(s \times(0,1))$ is clearly a capset of $\vec{Q}$, by the remarks preceding Proposition 2.1, $X=(s \times(0,1)) \cup(Q \times\{0,1\})$ is the complement of a capset in $\vec{Q}$, and hence is homeomorphic to $s$. We shall construct the required isotopy on $X$.

Observe that by (4), $G(X \times I)=X$. Consequently, $H=G \mid(X \times I)$ is an isotopy on $X$. Define $A=Q \times\{0\}$ and $B=Q \times\{1\}$, respectively. We shall prove that $H, A$ and $B$ satisfy (1) and (2). Of course, only (2) needs 
verification. To this end, let $E \subseteq X$ be closed such that $E \cap A=\varnothing$, and let $U$ be an open neighborhood of $B$ in $X$. Then $\bar{E} \cap A=\varnothing(\bar{E}$ is the closure of $E$ in $\vec{Q}$ ) because $A$ is compact. So there exists an element $t \in(0,1]$ with $\bar{E} \subseteq Q \times[t, 1]$. Let $U^{\prime} \subseteq \vec{Q}$ be open such that $U^{\prime} \cap X=U$. There is an element $s \in(0,1)$ such that $Q \times[s, 1] \subseteq U^{\prime}$. By (5) there exists $n \in \mathbf{N}$ with $\left(F_{1}\right)^{n}(t) \geq s$. Then clearly $\left(G_{1}\right)^{n}(\bar{E}) \subseteq U^{\prime}$, i.e. $\left(H_{1}\right)^{n}(E) \subseteq U$.

Now for $n \in \mathbf{N}$ let $x_{n}=(1,1 / n) \in \mathbf{R}^{2}$. The straight line segment connecting $(0,0)$ and $x_{n}$ will be denoted by $T_{n}$. Let $T$ denote the subspace $\bigcup_{n=1}^{\infty} T_{n}$ of $\mathbf{R}^{2}$. We define a homeomorphism $\xi: T \times s \rightarrow T \times s$ as follows. On $\{(0,0)\} \times s, \xi$ is the homeomorphism $H_{1}$ of Propostion 2.1 (we make an obvious identification here). For every $n, \xi \mid\left\{x_{n}\right\} \times s$ is the identity. We use the isotopy of Proposition 2.1 to "connect" $\xi \mid\{(0,0)\} \times s$ and $\xi \mid\left\{x_{n}\right\} \times s$ for every $n$. Clearly, the resulting function is a homeomorphism.

The space $T$ is contractible, locally contractible and 1-dimensional. Consequently, $T$ is an $\mathbf{A R}$ ([4]). Since $T$ is also topologically complete, by a theorem of Torunczyk [6], $T \times s$ is homeomorphic to $s$; let $h: s \rightarrow T \times s$ be any homeomorphism. Then

$$
\eta=h^{-1} \circ \xi \circ h: s \rightarrow s
$$

is a homeomorphism and we claim that it is as desired.

\subsection{Proposition. $\eta$ is not conjugate to an extendable homeomorphism.}

Proof. To the contrary, assume that there exists a homeomorphism $f: s \rightarrow s$ such that $f^{-1} \eta f=f^{-1} h^{-1} \xi h f$ is extendable to a homeomorphism $\rho: Q \rightarrow Q$. It will be convenient to let $g$ denote the composition $h \circ f: s \rightarrow T \times s$. Let $\underline{0}$ be the point in $s$ all coordinates of which are equal to 0 . For every $n$, let $p_{n}=g_{n}^{-1}\left(x_{n}, \underline{0}\right)$ and let $p \in Q$ be a limit point of the sequence $\left(p_{n}\right)_{n}$. Observe that $\left\{p_{n}: n \in \mathbf{N}\right\}$ is closed in $s$ so that $p \notin s$. Also observe that every $p_{n}$ is a fixed point of $g^{-1} \xi g$ from which it follows that $p$ is a fixed point of $\rho$.

Claim. $p \notin \overline{g^{-1}(\{\underline{0}\} \times s)}$.

To the contrary, assume that $p \in \overline{g^{-1}(\{\underline{0}\} \times s)}$. Since $A$ and $B$ are compact and $p \notin g^{-1}(\{\underline{0}\} \times s)$ there are open neighborhoods $U$ and $V$ of $A$ and $B$ in $s$, respectively, such that

$$
p \notin \overline{g^{-1}(\{\underline{0}\} \times(U \cup V))} .
$$

Put $E=s \backslash(U \cup V)$. Then $E$ is closed in $s$, misses $A$, and clearly has the property that $p \in \overline{g^{-1}(\{\underline{0}\} \times E)}$. By Proposition 2.1 there exists $n \in \mathbf{N}$ such that $\left(H_{1}\right)^{n}(E) \subset V$. This implies that

$$
\begin{aligned}
\left(g^{-1} \xi g\right)^{n}\left(g^{-1}(\{\underline{0}\} \times E)\right) & =g^{-1} \xi^{n} g\left(g^{-1}(\{\underline{0}\} \times E)\right) \\
& =g^{-1} \xi^{n}(\{\underline{0}\} \times E) \subseteq g^{-1}(\{\underline{0}\} \times V) .
\end{aligned}
$$


Consequently,

$$
\rho^{n}(p) \in \overline{\rho^{n}\left(g^{-1}(\{\underline{0}\} \times E)\right)} \subseteq \overline{g^{-1}(\{\underline{0}\} \times V)} \subseteq Q \backslash\{p\} .
$$

But this contradicts the fact that $p$ is a fixed point of $\rho$.

By the claim there is an open neighborhood $U$ of $p$ in $Q$ such that

(1) $U \cap\left(g^{-1}(\{\underline{0}\} \times s)\right)=\varnothing$;

(2) $U \cap s$ is connected

(simply let $U$ be a basic open subcube of $Q$ containing $p$ but missing $\left.g^{-1}(\{\underline{0}\} \times s)\right)$. Observe that $U \cap s$ contains infinitely many $p_{n}$ 's. Consequently, $g(U \cap s)$ is a connected open subset of $T \times s$ which misses $\{\underline{0}\} \times s$ and contains infinitely many $\left(x_{n}, \underline{0}\right)$ 's. This is clearly a contradiction.

2.3. Question. Is every homeomorphism on $s$ the composition of two conjugates of extendable homeomorphisms?

\section{REFERENCES}

1. R. D. Anderson and R. H. Bing, A complete elementary proof that Hilbert space is homeomorphic to the countable product of lines, Bull. Amer. Math. Soc. 74 (1968), 771-792.

2. C. Bessaga and A. Pelczyński, Selected topics in infinite-dimensional topology, Polish Sci. Publ., Warsaw, 1975.

3. T. A. Chapman, Dense sigma-compact subset of infinite-dimensional manifolds, Trans. Amer. Math. Soc. 154 (1971), 399-426.

4. S. T. Hu, The theory of retracts, Wayne State University Press, 1965.

5. K. Sakai and R. Y. T. Wong, Conjugating homeomorphisms to uniform homeomorphisms, Trans. Amer. Math. Soc. (to appear).

6. H. Torunczyk, Absolute retracts as factors of normed linear spaces, Fund. Math. 86 (1974), 53-67.

Faculteit Wiskunde en Informatica, Vrije Universiteit, De Boelelaan 1081, 1081 hV AMSTERDAM, THE NetherLANDS

Faculteit Wiskunde en Informatica, Universiteit VAN AMSTERdaM, Roeters StraAt 15, 1018 WB Amsterdam, The Netherlands 